\title{
Antibiotic Sensitivity Pattern and Plasmid Profile Of Urinary Tract Infection Isolates among Children Below 10 Years Of Age
}

\author{
${ }^{1}$ Ram Wagle, ${ }^{1}$ Rajendra Timilsina, ${ }^{1}$ Rojesh Thapa, ${ }^{2}$ Nabaraj Adhikari, ${ }^{2}$ Upendra Thapa \\ Shrestha, ${ }^{1}$ Suresh Jaiswal, ${ }^{1}$ Bishnu Raj Tiwari,
}

${ }^{1}$ School of Health and Allied Sciences, Pokhara University, Kaski, Nepal

${ }^{2}$ Kantipur College of Medical Sciences, Sitapaila, Kathmandu, Nepal

\begin{abstract}
Urinary tract infection (UTI) is defined as colonization of pathogen anywhere along the urinary tract. UTI has been classified by site of infection as Upper urinary tract infection and lower urinary tract infection and by severity as Complicated and uncomplicated UTI. This community based cross sectional study was conducted to determine the Antibiotic sensitivity pattern and plasmid profile of most prevalent urinary tract infection isolates among children below 10 years of age, from December 2013 to February 2014. Among the total 800 sample collected from the community 390(48.8\%) were female and 410(51.2\%) were male. The prevalence rate was found to be $44(5.5 \%)$ of total cases and was statistically significant $(p<0.05)$. Among significant growth, $15(1.9 \%)$ and $29(3.6 \%)$ were male and female respectively $(p<0.05)$. Out of 44 total isolates frequency of Escherichia coli (E. coli) was 20 followed by Staphylococcus aureus(12), which accounted for $45.5 \%$ and $27.3 \%$ respectively. Remaining were Pseudomonas(11.4\%), Klebsiella(11.4\%), Proteus(2.3\%)and Citrobacter sps.(2.3\%). Tobramycin(100\%) and Amikacin(97.73\%) were found to be the most sensitive antibiotics followed by Chloramphenicol(93.18\%), Imipenem(90.91\%) and Ciprofloxacin(75\%) respectively.Out of 20 E. coli isolates, no plasmid was seen in $7(35 \%)$ while $8(40 \%)$ showed single plasmid which was present in 8 isolates. Plasmid copy number of 2, 3 and 4 were displayed by 2(10\%), 2(10\%) and 1(5\%) of the isolates respectively. A common (>21 kilobases) plasmid was the most common among isolates under study. This study revealed that E. coli was the most prevalent organism causing community acquired pediatric UTI. Antibiotics that are commonly used for the management of UTI and other cases are being more resistant i.e., Ampicillin. Plasmid analysis showed the presence of plasmids in resistant E. coli isolates that might harbor resistant genes. So that further analysis is required for the detection of responsible genes.
\end{abstract}

Key words: Antibiotic sensitivity pattern, Plasmid profile, Urinary tract infection

Corresponding author: School of Health and Allied Sciences, Pokhara University, Kaski, Nepal.

E-mail: bishnurajtiwari@gmail.com

\section{INTRODUCTION}

Urinary tract infection (UTI) is defined as colonization of pathogen anywhere along the urinary tract. In children more simpler and practical approach of categorization of UTI as a first time infection versus recurrent infection. Recurrent infections can be further subdivided into unresolved bacteriuria, bacterial persistence, and re-infection. The recurrence of a UTI may be caused by several reasons. Unresolved bacteriuria is most commonly caused by inadequate antimicrobial therapy. Sub-therapeutic levels of the antimicrobial agents may be a result of noncompliance, mal-absorption, suboptimal drug metabolism, and resistant uropathogens unresponsive to attempted therapy. ${ }^{1}$ In these cases, infection typically resolves after altering the therapy according to antimicrobial sensitivities determined by a proper urine culture. In the case of bacterial persistence, the nidus of infection in the urinary tract is not eradicated. Characteristically, the same pathogen is documented on urine cultures during subsequent episodes of UTI despite negative cultures after treatment. Re-infection is characterized by different pathogens documented on proper urine cultures with each new UTI, sometimes with same microorganism, which may have persisted in the vagina or feces create a new infection.

The true incidence of pediatric UTI is difficult to determine because there are varying presentations that range from an absence of specific urinary complaints to fulminant urosepsis. Urinary tract infection (UTI) occurs in approximately $8 \%$ of girls and $2 \%$ of boys by 7 years of age. The frequency of symptomatic UTI during the first 10 years of life is $3.0 \%$ in girls and $1.1 \%$ in boys. ${ }^{2}$

Microbial etiology of UTIs has been regarded as well as established, with $E$. coli being the causative pathogen in $50-80 \%$ of cases and other Enterobacteriaceae (Klebsiella, Proteus, Enterobacter) together with Enterococci, Streptococci, Staphylococci, and Pseudomonas sps account for most of the remaining positive urine cultures of children. ${ }^{3}$ Children who have UTI do not necessarily present with the characteristic signs and symptoms although various clinical presentation includes fever, flank pain, dysuria, oligouria, 
Wagle et. al. Antibiotic Sensitivity Pattern and Plasmid Profile Of Urinary Tract ....., JHAS, 2016, Vol. 5, No. 1 P 58-62

abdominal discomfort, anorexia, nausea and vomiting.

Female gender, social concerns, Infants, foreskin, fecal and perineal colonization, Urinary tract anomalies, Functional abnormalities, immune-compromised state, Sexual activity are regarded as the risk factors for urinary tract infection in children.

Bacteria enter into the urinary tract by the fecal-perinealurethral route with subsequent attachment, colonization and ascend to the bladder and once the uropathogens reaches the bladder, it may ascend to the ureters and then to the kidneys. Additional pathways of infection include, hematogenous seeding in the setting of systemic infection, lymphatic route and direct extension caused by the presence of fistulae from the bowel or vagina.

Antibiotic sensitivity is the susceptibility of bacteria to antibiotics. Antibiotic susceptibility testing (AST) is usually carried out to determine which antibiotic will be most successful in treating a bacterial infection in vivo. Commonly used methods of AST include diffusion, dilution and E-test. Antibiotic resistance is the ability of a microorganism to withstand the effects of an antibiotic. It is one of the major causes of failure in the treatment of infectious diseases that results in increased morbidity, mortality, and costs of health care. Factors contributing towards resistance include incorrect diagnosis, unnecessary prescriptions, improper use of antibiotics by patients, and the use of antibiotics as livestock food additives for growth promotion. ${ }^{4}$

Plasmids are defined as double stranded, extra-chromosomal genetic elements that replicate independently of the host cell chromosome and are stably inherited that may encode products(protein) that aid in virulence, pathogenicity, and the spread of resistance among a wide spectrum of bacteria. Plasmids differ from chromosomes in being small and coding for genes that are non-essential for the bacterial survival. Absence of plasmids doesn't kill bacterium, but their presence provides additional benefits to the bacterial cell.

Urinary tract infection is the common and life threatening clinical condition which in case of community acquired infection usually remains undiagnosed. Untimely diagnosis and improper use of antibiotics for treatment of UTI results in re-current infection with subsequent resistant to the antibiotics commonly used in clinical practice. Antibiotic sensitivity of commonly isolated uropathogens and subsequent plasmid profile may correlate with the resistant pattern that helps for the detection of resistant genes and further drug discovery.

\section{MATERIAL AND METHODS}

A community based cross sectional descriptive study was carried out at School of Health and Allied Sciences, Pokhara University, Lekhnath-12, Kaski from December 2013 to
February 2014 and plasmid profiling was done at Kantipur College of Medical Sciences, Sitapaila, Kathmandu, Nepal. Sample was collected from various schools of Kaski and Tanahun district namely Shree Brahma Rupa Primary School, Shree Gaun Farka Primary School, Shree Himalayan Primary School, Shree Chandra Jyoti Primary School, Shree Bishow Shanti Primary School, Kaski, and Shree Pancha Jyoti primary School, Shree Basanta Kali Primary School, Tanahun. Eight hundred Clean catch Mid-Stream Urine (CCMSU) sample (at least $5 \mathrm{ml}$ ) was collected in sterile urine culture container from children below 10 years of age. Appropriate collection instructions were given at site and oral expressed consent was taken from the subjects. Sample was transported in ice pack and processed for isolation, identification and antimicrobial susceptibility testing using standard methodology and Plasmid isolation from Alkali lysis method. ${ }^{5}$

\section{RESULTS}

Table: 1 . Growth pattern among gender

\begin{tabular}{|c|c|c|c|c|}
\hline Growth & Frequency & Female & Male & Percent (\%) \\
\hline Insignificant & 756 & $361(45.1 \%)$ & $395(49.4 \%)$ & $94.5 \%$ \\
\hline Significant & 44 & $29(3.6 \%)$ & $15(1.9 \%)$ & $5.5 \%$ \\
\hline Total & 800 & $390(48.8 \%)$ & $410(51.2 \%)$ & $100.0 \%$ \\
\hline
\end{tabular}

Out of 800 urine sample collected from the community $756(94.5 \%)$ showed insignificant growth. Overall prevalence rate was found to be $5.5 \%$ i.e. 44 out of total cases and was statistically significant $(\mathrm{p}=0.000)$. Among which $15(1.9 \%)$ and $29(3.6 \%)$ were male and female respectively with male: female ratio of $1: 1.93(\mathrm{p}=0.019)$.

Table: 2. Overall distribution of AST pattern in different uropathogens

\begin{tabular}{|c|c|c|c|c|c|c|c|c|c|c|c|c|c|c|c|c|c|c|}
\hline \multirow{2}{*}{ 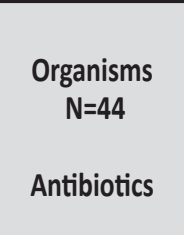 } & \multicolumn{3}{|c|}{ 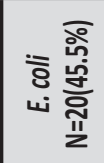 } & \multicolumn{3}{|c|}{ 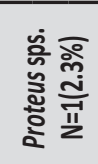 } & \multicolumn{3}{|c|}{ 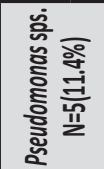 } & \multicolumn{3}{|c|}{ 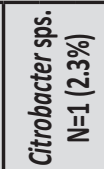 } & \multicolumn{3}{|c|}{ 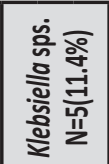 } & \multicolumn{3}{|c|}{ 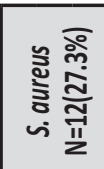 } \\
\hline & s & & & $S$ & & & $S$ & & $\mathrm{R}$ & $s$ & & & & & R & & & $R$ \\
\hline & & 0 & 0 & & & & 0 & & & 0 & & & 0 & & & & 0 & 12 \\
\hline & & 0 & U & 1 & 0 & & 5 & & 0 & 1 & 0 & 0 & & 1 & 0 & & 0 & 0 \\
\hline proflo & & 0 & 7 & 1 & 0 & 0 & 4 & & & 1 & 0 & 0 & 4 & 1 & 0 & 10 & 1 & 1 \\
\hline & & & & 1 & 0 & & 2 & & & 1 & v & & & 0 & 0 & & 0 & 0 \\
\hline IIp & & 3 & 1 & 1 & 0 & 0 & 5 & 0 & 0 & 1 & 0 & 0 & 5 & 0 & 0 & 12 & 0 & 0 \\
\hline d & 17 & 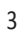 & 0 & 0 & 0 & 1 & 0 & & & 1 & 0 & 0 & 1 & 2 & 2 & 12 & 0 & 0 \\
\hline lid & 8 & 1 & 11 & 0 & 0 & 1 & 0 & 2 & 3 & 0 & 0 & 1 & 3 & 2 & 0 & 0 & 0 & 12 \\
\hline & 0 & 0 & 20 & 0 & 0 & & 0 & & 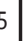 & 0 & v & 1 & & 0 & 5 & 8 & 4 & 0 \\
\hline bra & 20 & 0 & & 1 & 0 & & 5 & & U & 0 & 1 & 0 & & 0 & 0 & & 0 & 0 \\
\hline Cor & 0 & 6 & 14 & 0 & 0 & & 0 & & 0 & 0 & 1 & 0 & & 3 & 1 & 0 & 2 & 10 \\
\hline Ceftazidime & 0 & 0 & 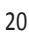 & 0 & 0 & 1 & 0 & & 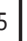 & 0 & 0 & 1 & 0 & 0 & 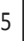 & 0 & 0 & 12 \\
\hline & 0 & 5 & 5 & 1 & 0 & & 1 & & 1 & 0 & 1 & 0 & & 3 & 2 & 9 & 3 & 0 \\
\hline trimoxazole & 14 & 0 & P & 01 & 0 & & 0 & 2 & 3 & 1 & 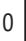 & 0 & & 0 & 2 & & 2 & 2 \\
\hline
\end{tabular}


(Note: $-\mathrm{S}=$ sensitive, $\mathrm{I}=$ intermediate, $\mathrm{R}=$ resistant)

Among 44 total isolates frequency of $E$. coli was 20 followed by $S$. aureus (12), which accounted for $45.5 \%$ and $27.3 \%$ respectively. Remaining were Pseudomonas, Klebsiella, Proteus and Citrobacter sps. Most sensitive drugs were Tobramycin (100\%), Amikacin (97.73\%), Chloramphenicol (93.18\%) and Imepenem (90.91\%) while most resistant drugs were Ampicillin (100\%), Ceftazidime (100\%), Vancomycin $(72.73 \%)$ and cefotaxime $(70.45 \%)$.

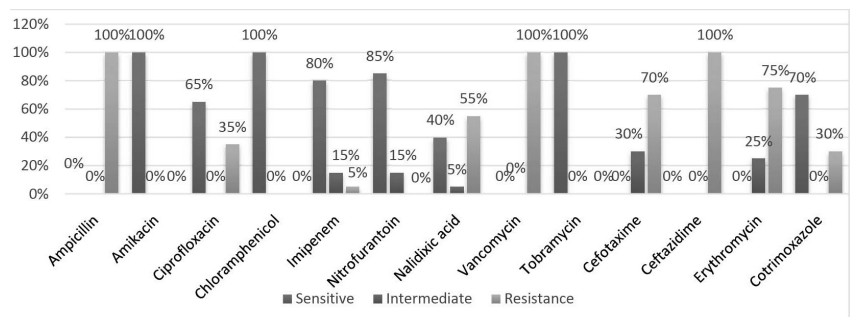

Figure: 1 Antibiotic Sensitivity pattern of $E$. coli

All E. coli isolates (20) were $100 \%$ sensitive to Amikacin, Chloramphenicol and Tobramycin followed by Nitrofurantoin (85\%), Imepenem (80\%) and Cotrimoxazole (70\%). Ampicillin, Vancomycin, and Ceftazidime were 100\% resistant followed by Erythromycin (75\%), Cefotaxime $(70 \%)$ and Nalidixic acid (55\%).

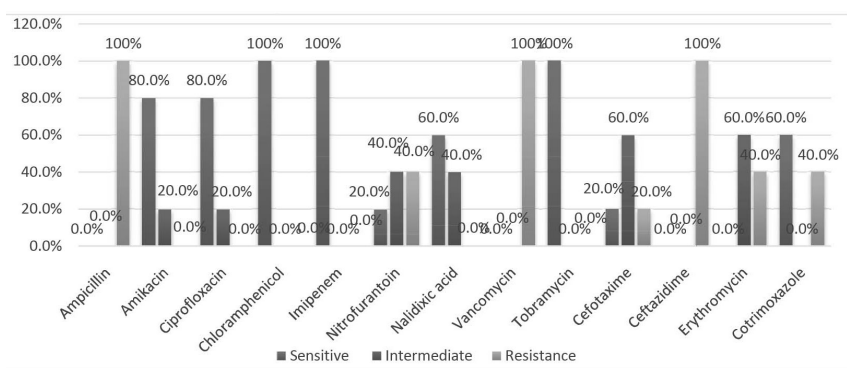

Figure: 2 Antibiotic sensitivity pattern of Klebsiella sps.

All Klebsiella sps. isolates were $100 \%$ sensitive to Chloramphenicol, Imepenem and Tobramycin followed by Amikacin (80\%) and Ciprofloxacin (80\%). Ampicillin, Vancomycin and Ceftazidime were 100\% resistant followed by Cotrimoxazole (40\%), Erythromycin $(40 \%)$ and Nitrofurantoin $(40 \%)$.

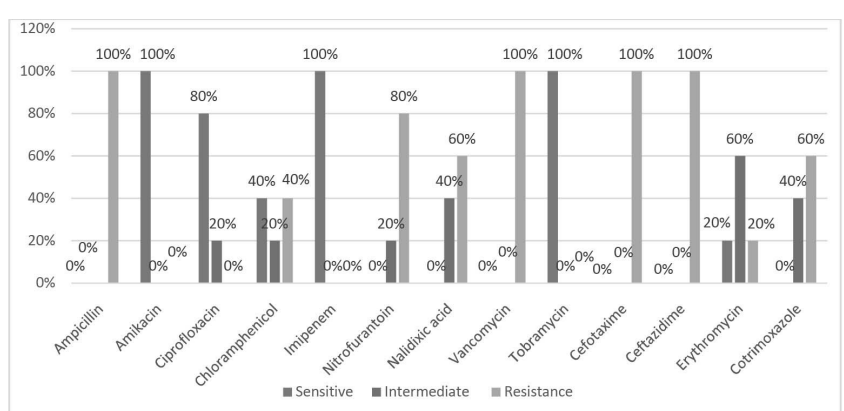

Figure: 3 Antibiotic Sensitivity patterns of Pseudomonas sps.

All Pseudomonas sps. isolates were $100 \%$ sensitive to Amikacin, Imepenem and Tobramycin followed by Ciprofloxacin (80\%). Ampicillin, Vancomycin, Ceftazidime and Cefotaxime were 100\% resistant followed by Nitrofurantoin (80\%), Nalidixic acid $(60 \%)$ and Cotrimoxazole $(60 \%)$.

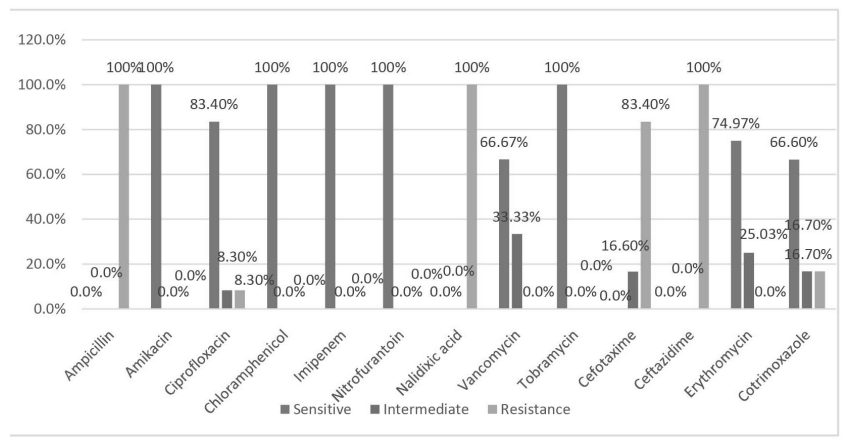

Figure: 4 Antibiotic Sensitivity pattern of $S$. aureus.

All S. aureus isolates were $100 \%$ sensitive to Amikacin, Chloramphenicol, Imepenem, Nitrofurantoin and Tobramycin followed by Ciprofloxacin (83.4\%), Erythromycin (74.97\%) and Vancomycin (66.67\%). Ampicillin, Nalidixic acid and Ceftazidime were $100 \%$ resistant followed by Cefotaxime (83.4\%).

Table 3: Plasmid copy number and prevalence among total $E$. coli isolates $(\mathrm{N}=\mathbf{2 0})$.

\begin{tabular}{|c|c|c|}
\hline $\begin{array}{c}\text { Plasmid } \\
\text { copy number }\end{array}$ & $\begin{array}{c}\text { No. of isolates } \\
(\mathrm{N}=20)\end{array}$ & prevalence \\
\hline 0 & $\mathbf{7}$ & $\mathbf{3 5 \%}$ \\
\hline 1 & $\mathbf{8}$ & $40 \%$ \\
\hline 2 & $\mathbf{2}$ & $10 \%$ \\
\hline 3 & $\mathbf{2}$ & $10 \%$ \\
\hline 4 & 1 & $\mathbf{5 \%}$ \\
\hline
\end{tabular}

Among the total E. coli isolates, no plasmid was seen in $7(35 \%)$ while $40 \%$ showed single plasmid which was present in 8 isolates. Plasmid copy number of 2, 3 and 4 were displayed by $2(10 \%), 2(10 \%)$ and $1(5 \%)$ of the isolates respectively.

Electrophoretic pattern of plasmid DNA of $E$. coli isolates Lane 1 Lamda/EcoR1

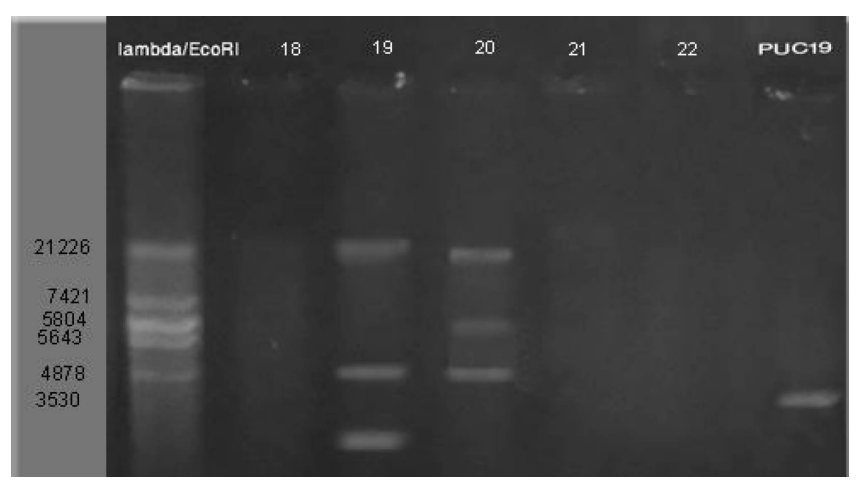

(Note: - Lane 1= Lamda/EcoR1 ladder, Lane 17= pUC19 
control DNA (2.6 kb), lane 2-22 E. coli plasmid DNA). Plasmid copy number of 0 to 4 were seen among E. coli isolates with different molecular size $(0$ to $>21 \mathrm{~kb})$. All $E$. coli having single copy number showed $>21 \mathrm{~kb}$ plasmid that were resistant to 4-6 antibiotics used.

Table: 4 Relation of AST with Plasmid profile of $E$. coli isolates

\begin{tabular}{|l|l|l|l|}
\hline \multicolumn{1}{|c|}{ Resistant drugs } & $\begin{array}{c}\text { Lane } \\
\text { no. }\end{array}$ & $\begin{array}{c}\text { Plasmid } \\
\text { copy number }\end{array}$ & \multicolumn{1}{|c|}{$\begin{array}{c}\text { Molecular } \\
\text { weight(MW) }\end{array}$} \\
\hline AMP,VA,NA,CAZ,E & 2 & 1 & $>21 \mathrm{~kb}$ \\
\hline AMP,VA,CTX,CAZ,E & 3 & 1 & $>21 \mathrm{~kb}$ \\
\hline $\begin{array}{l}\text { AMP, IPM, CIP,VA,NA, } \\
\text { TX,CAZ,E,CO }\end{array}$ & 4 & 4 & $\begin{array}{l}>21 \mathrm{~kb}, 18 \mathrm{~kb}, \\
6.5 \mathrm{~kb}, 5.8 \mathrm{~kb}\end{array}$ \\
\hline AMP,NA,VA,CTX,CAZ,E & 5 & 1 & $>21 \mathrm{~kb}$ \\
\hline AMP,VA,CAZ & 6 & 0 & \\
\hline AMP,VA,CAZ,E & 7 & 0 & \\
\hline AMP,VA,NA,CAZ,E & 8 & 1 & $>21 \mathrm{~kb}$ \\
\hline AMP,NA,VA,CAZ,E,CTX,CO & 9 & 2 & $>21 \mathrm{~kb},>21 \mathrm{~kb}$ \\
\hline AMP,VA,CAZ,CTX,E & 10 & 0 & \\
\hline AMP,NA,VA,CAZ,E,CTX,CO & 11 & 2 & $>21 \mathrm{~kb}, 14.5 \mathrm{~kb}$ \\
\hline AMP,VA,CTX,CAZ & 12 & 1 & $>21 \mathrm{~kb}$ \\
\hline AMP,VA,CTX,CAZ,CO,E & 13 & 1 & $>21 \mathrm{~kb}$ \\
\hline AMP,VA,CAZ,CTX,E & 14 & 1 & $>21 \mathrm{~kb}$ \\
\hline AMP,CIP,NA,VA,CAZ,CTX & 15 & 0 & \\
\hline AMP,CIP,NA,VA,CAZ,CTX,E & 16 & 1 & $>21 \mathrm{~kb}$ \\
\hline AMP,CIP,VA,CAZ,CTX & 18 & 0 & \\
\hline AMP,CIP,NA,VA,CTX,CAZ,E,CO & 19 & 3 & $>21 \mathrm{~kb}, 4.8 \mathrm{~kb},<2.6 \mathrm{~kb}$ \\
\hline AMP,CIP,NA,VA,CTX,CAZ,E,CO & 20 & 3 & $17.5 \mathrm{~kb}, 5.4 \mathrm{~kb},<4.8 \mathrm{~kb}$ \\
\hline AMP,CIP,NA,VA,CAZ & 21 & 0 & \\
\hline AMP,VA,CAZ,E & 22 & 0 & \\
\hline
\end{tabular}

Note :-AMP(Ampicillin), CTX(Cefotaxime),

CAZ(Ceftazidime), CIP(Ciprofloxacin), $\mathrm{CO}(\mathrm{Co}-$

trimoxazole), E(Erythromycin), IPM(Imepenem),

NA(Nalidixic acid), NIT( Nitrofurantoin) and

VA(Vancomycin).

\section{DISCUSSION}

It was a community based cross sectional descriptive study. Sample was collected from various schools of Kaski and Tanahun district. Overall prevalence rate was found to be (5.5\%) 44 out of total cases (800) and was statistically significant $(\mathrm{p}<0.05)$. Male: female ratio of $1: 1.93(\mathrm{p}<0.05)$. Female cases were high as compared to male cases because short and close proximity of anus to urethra, relatively high moisture and socio-economic concerns determines the outcome of infection.

In this study prevalence of $E$. coli was higher (45.5\%) as compared to other isolates from community acquired UTI. Ghadage et al., also showed that $E$. coli was present in $45.12 \%$ of total isolates. ${ }^{6}$ Similar research done by Gautam et al., at Gandaki Medical College Teaching Hospital, Pokhara,
Nepal showed slightly lower (39.4\%) percentage of E. coli. Overall sensitivity pattern against all isolates were Tobramycin (100\%), Amikacin (97.73\%), Chloramphenicol (93.18\%) and Imepenem (90.91\%) while most resistant were Ampicillin (100\%), Ceftazidime (100\%), Vancomycin $(72.73 \%)$ and cefotaxime $(70.45 \%)$. Antibiotic sensitivity pattern is different and changing among local population, nationally and globally. Previous results from Paudel et al., have shown $94 \%$ sensitivity to Amikacin. ${ }^{8}$ In the study carried out by Forouzan et al., Ampicillin resistance rate was more than $90 \% .{ }^{9}$

All E. coli isolates (20) were $100 \%$ sensitive to Amikacin, Chloramphenicol and Tobramycin followed by Nitrofurantoin (85\%), Imepenem $(80 \%)$ and Cotrimoxazole (70\%). Ampicillin, Vancomycin, and Ceftazidime were 100\% resistant followed by Erythromycin (75\%), Cefotaxime $(70 \%)$ and Nalidixic acid (55\%). Increased resistance to third generation cephalosporins might be due to ESBL production. Acharya et al., also reported $100 \%$ ampicillin resistance to uropathogenic E. coli from Bharatpur, Nepal. ${ }^{10}$

Antibiotics that were effective against Klebsiella sps in our study were Chloramphenicol, Tobramycin, Imepenem and Amikacin supported by previous study results. ${ }^{11}$ However Cotrimoxazole and Nitrofurantoin showed moderate levels of resistance same as for Pseudomonas except for Nitrofurantoin which was higher $(80 \%)$.

All S. aureus isolates were $100 \%$ sensitive to Amikacin, Chloramphenicol, Imepenem, Nitrofurantoin and Tobramycin followed by Ciprofloxacin (83.4\%) whereas Vancomycin was $66.67 \%$ sensitive (33.33\% were intermediate). Paudel et al., also showed $100 \%$ sensitivity to Amikacin followed by Ciprofloxacin (96\%).

Among the total E. coli isolates, no plasmid was seen in $7(35 \%)$ while plasmids were present in $13(65 \%)$. Single plasmid was present in $8(40 \%)$ isolates while plasmid copy number of 2, 3 and 4 were displayed by $2(10 \%), 2(10 \%)$ and $1(5 \%)$ of the isolates respectively. Plasmid copy number of 0 to 4 were seen among $E$. coli isolates with different molecular size approximately $(0$ to $>21 \mathrm{~kb})$. All $E$. coli having single copy number showed $>21 \mathrm{~kb}$ plasmid which was the most prevalent one, that were resistant to 4-6 antibiotics used. In a study carried out by khadgi et al., a band of approximately $23 \mathrm{~kb}$ was seen in most of the $E$. coli isolates. ${ }^{12}$ In our study strains showing 2 copy number were resistant to same class of antibiotics but different plasmid profile was seen among which one strain showed two $>21 \mathrm{~kb}$ plasmids while $>21 \mathrm{~kb}$ and $14.5 \mathrm{~kb}$ plasmids were seen in another strain. Isolates showing 3 plasmid copy number were resistant to same eight antibiotics used but different profiles were observed. For isolate in which 4 plasmid copy number was present, $>21 \mathrm{~kb}, 18 \mathrm{~kb}, 6.5 \mathrm{~kb}$ and $5.8 \mathrm{~kb}$ plasmids were seen which was resistant 9 antibiotics under study. According to Sadeghi et $a l$., on their study "Plasmid profile of E. coli strain isolated 
from UTI of inpatient and outpatient". Ten strains (10\%) lacked any plasmid and in 90 strains (90\%), 1-7 plasmids were detected. Although some strains contained plasmids of $>21 \mathrm{~kb}$ molecular mass, but mainly isolated plasmids ranged from $0.9 \mathrm{~kb}$ to $21 \mathrm{~kb} .{ }^{13}$

Our study suggests that $E$. coli is the predominant cause of community acquired pediatric UTI.
Antibiotics that are commonly used for the management of UTI cases are being less effective i.e., Ampicillin. Plasmid analysis showed the presence of plasmids in resistant $E$. coli isolates that might harbor resistant genes. So that further analysis of R-plasmid is required for the detection of responsible genes.

\section{ACKNOWLEDGEMENT}

We would like to acknowledge to the School of Health and Allied Sciences for his management and laboratory support during the research period. We would like to show our greatest appreciation to family of Shree Brahma Rupa Primary School, Shree Gaun Farka Primary School, Shree Himalayan Primary School, Lekhnath-13-TalloGagangauda, Shree Chandra Jyoti Primary School, lekhnath-15, Dhodbesi, Shree Bishow Shanti Primary School, Kaski and Shree Pancha Jyoti primary School, Shree Basanta Kali Primary School, Tanahun, for help and co-operation in sample collection process. We are thankful to Kantipur College of Medical Sciences, Kathmandu for co-operation and technical guidance during plasmid profiling.

\section{REFERENCES}

1. Pewitt EB, Schaeffer AJ. Urinary Tract Infection in urology, including acute and chronic prostatitis. Infect Dis Clin North Am, 1997; 11(3):623- 46.

2. Winberg J, Bergstrom T, Jacobsson B. Morbidity, age and sex distribution, recurrences and renal scarring in symptomatic Urinary Tract Infection in childhood. Kidney Int. Suppl, 1975; 4:S101-6.

3. Kahlmeter G. An international survey of the Antimicrobial Susceptibility of pathogens from uncomplicated Urinary Tract Infections: The ECO-SENS project. J Antimicrob Chemother, 2003; 51(1):69-76.

4. Bouza E, Cercenado E. Klebsiella and Enterobacter: Antibiotic resistance and treatment implications. Semin Respir Infect, 2002; 17:215-230

5. Vandepitte J, Verhaegen J, Engbaek K, Rohner P, Piot P, Heuck CC. Basic laboratory proceduresin clinical bacteriology. WHO, 2003.

6. Kashef N, Djavid GE, Shahbazi S. Antimicrobial susceptibility patterns of community-acquired uropathogens in Tehran, Iran. JIDC, 2010; 4(4):202-6.

7. Dromigny JA, Perrier NP, Gros JD. Claude Distribution and susceptibility of bacterial urinary tract infections in Dakar, Senegal. Int. J Antimicrob Agents, 2002; 20:339-47.

8. Daza R, Piedrola GJ. Antibiotic susceptibility of bacterial strains isolated from with community-acquired urinary tract infections. Int. J Antimicrob Agents, 2001;18:211-15.

9. Lau SM, Chang PM. Resistance rates to commonly used antimicrobials among pathogens of both bacteremic and nonbacteremic community-acquired urinary tract infection. J Microbiol Immunol Infect, 2004; 37:185-91.

10. Acharya A, Gautam R, Subedee L. Uropathogens and their antimicrobial susceptibility pattern in Bharatpur, Nepal. Nepal Med Coll J, 2011; 13(1): 30-33.

11. Sternheimer R, Malbin B. Clinical recognition of pyelonephritis with a new strain for Urinary Sediments. Am J Med, 1951;11(3):312-23.

12. Paudel K, Gurung NS, Paudel B. A study on the sensitivity pattern of UTI pathogens in CHRC Pokhara. Journal of Gandaki Medical College. Nepal, 2010; 3:1.

13. Pape L, Gunzer F, Ziesing S, Bacterial pathogens, resistance patterns and treatment options in community acquired pediatric urinary tract infection. Klin Padiatr, 2004; 216(2):83-6. 